Louisiana State University

LSU Digital Commons

$1-1-1996$

\title{
The russian-american gallium experiment (sage) cr neutrino source measurement
}

\author{
J. N. Abdurashitov \\ Institute for Nuclear Research of the Russian Academy of Sciences \\ V. N. Gavrin \\ Institute for Nuclear Research of the Russian Academy of Sciences \\ S. V. Girin \\ Institute for Nuclear Research of the Russian Academy of Sciences \\ V. V. Gorbachev \\ Institute for Nuclear Research of the Russian Academy of Sciences \\ T. V. Ibragimova \\ Institute for Nuclear Research of the Russian Academy of Sciences
}

See next page for additional authors

Follow this and additional works at: https://digitalcommons.Isu.edu/physics_astronomy_pubs

\section{Recommended Citation}

Abdurashitov, J., Gavrin, V., Girin, S., Gorbachev, V., Ibragimova, T., Kalikhov, A., Khairnasov, N., Knodel, T., Kornoukhov, V., Mirmov, I., Shikhin, A., Veretenkin, E., Vermul, V., Yants, V., Zatsepin, G., Bowles, T., Nico, J., Teasdale, W., Wark, D., Cherry, M., Karaulov, V., Levitin, V., Maev, V., Nazarenko, P., Shkol-Nik, V., Skorikov, N., Cleveland, B., Daily, T., Davis, R., Lande, K., Lee, C., Wildenhain, P., \& Khomyakov, Y. (1996). The russianamerican gallium experiment (sage) cr neutrino source measurement. Physical Review Letters, 77 (23), 4708-4711. https://doi.org/10.1103/PhysRevLett.77.4708

This Article is brought to you for free and open access by the Department of Physics \& Astronomy at LSU Digital Commons. It has been accepted for inclusion in Faculty Publications by an authorized administrator of LSU Digital Commons. For more information, please contact ir@lsu.edu. 


\section{Authors}

J. N. Abdurashitov, V. N. Gavrin, S. V. Girin, V. V. Gorbachev, T. V. Ibragimova, A. V. Kalikhov, N. G. Khairnasov, T. V. Knodel, V. N. Kornoukhov, I. N. Mirmov, A. A. Shikhin, E. P. Veretenkin, V. M. Vermul, V. E. Yants, G. T. Zatsepin, T. J. Bowles, J. S. Nico, W. A. Teasdale, D. L. Wark, M. L. Cherry, V. N. Karaulov, V. L. Levitin, V. I. Maev, P. I. Nazarenko, V. S. Shkol-Nik, N. V. Skorikov, B. T. Cleveland, T. Daily, R. Davis, K. Lande, C. K. Lee, P. W. Wildenhain, and Y. U.S. Khomyakov 


\section{Sussex Research Online}

\section{The Russian-American gallium experiment (SAGE) $\mathrm{Cr}$ neutrino source measurement}

Article (Published Version)

Wark, David and et al, (1996) The Russian-American gallium experiment (SAGE) Cr neutrino source measurement. Physical Review Letters, 77. pp. 4708-4711.

This version is available from Sussex Research Online: http://sro.sussex.ac.uk/id/eprint/24400/

This document is made available in accordance with publisher policies and may differ from the published version or from the version of record. If you wish to cite this item you are advised to consult the publisher's version. Please see the URL above for details on accessing the published version.

\section{Copyright and reuse:}

Sussex Research Online is a digital repository of the research output of the University.

Copyright and all moral rights to the version of the paper presented here belong to the individual author(s) and/or other copyright owners. To the extent reasonable and practicable, the material made available in SRO has been checked for eligibility before being made available.

Copies of full text items generally can be reproduced, displayed or performed and given to third parties in any format or medium for personal research or study, educational, or not-for-profit purposes without prior permission or charge, provided that the authors, title and full bibliographic details are credited, a hyperlink and/or URL is given for the original metadata page and the content is not changed in any way. 


\title{
The Russian-American Gallium Experiment (SAGE) Cr Neutrino Source Measurement
}

\author{
J. N. Abdurashitov, V. N. Gavrin, S. V. Girin, V. V. Gorbachev, T. V. Ibragimova, A. V. Kalikhov, N. G. Khairnasov, \\ T. V. Knodel, V. N. Kornoukhov,* I. N. Mirmov, A. A. Shikhin, E. P. Veretenkin, V. M. Vermul, \\ V.E. Yants, and G. T. Zatsepin \\ Institute for Nuclear Research, Russian Academy of Sciences, 117312 Moscow, Russia
}

T. J. Bowles, J. S. Nico, ${ }^{\dagger}$ W. A. Teasdale, and D. L. Wark ${ }^{\ddagger}$ Los Alamos National Laboratory, Los Alamos, New Mexico 87545

M. L. Cherry

Louisiana State University, Baton Rouge, Louisiana 70803

\author{
V. N. Karaulov, V. L. Levitin, V. I. Maev, P. I. Nazarenko, V. S. Shkol'nik, and N. V. Skorikov \\ Mangyshlak Atomic Energy Complex, Aktau, Republic of Kazakhstan
}

B. T. Cleveland, T. Daily, R. Davis, Jr., K. Lande, C. K. Lee, and P. W. Wildenhain

University of Pennsylvania, Philadelphia, Pennsylvania 19104

\author{
Yu. S. Khomyakov and A. V. Zvonarev \\ Power Physics Institute, Obninsk, Russia \\ S. R. Elliott and J.F. Wilkerson \\ University of Washington, Seattle, Washington 98195
}

(Received 22 August 1996)

\begin{abstract}
The solar neutrino capture rate measured by SAGE is well below that predicted by solar models. To check the overall experimental efficiency, we exposed 13 tonnes of Ga metal to a reactorproduced $517 \mathrm{kCi}$ source of ${ }^{51} \mathrm{Cr}$. The ratio of the measured production rate to that predicted from the source activity is $0.95 \pm 0.11$ (stat) $+0.05 /-0.08$ (syst). This agreement verifies that the experimental efficiency is measured correctly, establishes that there are no unknown systematic errors at the $10 \%$ level, and provides considerable evidence for the reliability of the solar neutrino measurement. [S0031-9007(96)01782-6]

PACS numbers: 26.65.+t, 95.85.Ry
\end{abstract}

The low threshold energy (232 keV [1]) for inverse beta decay on ${ }^{71} \mathrm{Ga}$ is well below the end point energy of the neutrino spectrum from proton-proton fusion. A Ga experiment can thus observe this low-energy branch of the solar neutrino spectrum. The Russian-American Gallium Experiment (SAGE) has measured a ${ }^{71} \mathrm{Ge}$ production rate of $69 \pm 10$ (stat) $+5 /-7$ (syst) SNU [2] (where $1 \mathrm{SNU}=$ $10^{-36}$ interactions/target atom/s), a value that is well below solar model predictions of $137+8 /-7 \mathrm{SNU}$ [3] or $125 \pm 5 \mathrm{SNU}$ [4]. Another Ga experiment, the GALLEX Collaboration, also observes a low rate of $69.7 \pm 6.7$ (stat) $+3.9 /-4.5$ (syst) SNU [5]. Taken together with other solar neutrino experimental results [6,7], a contradiction arises which cannot be accommodated by current solar models [8]. The significance of this conclusion requires that all possible instrumental checks of these experiments be performed. A test of the entire operation of the SAGE detector (i.e., the chemical extraction efficiency, counting efficiency, and analysis technique) with a known flux of low-energy neutrinos makes a rigorous check on the entire experiment.
${ }^{51} \mathrm{Cr}$ is a particularly good isotope to employ for this purpose $[9,10]$. The decay of ${ }^{51} \mathrm{Cr}$ is via electron capture to ${ }^{51} \mathrm{~V}$ with neutrino energies of $751 \mathrm{keV}(90.12 \%)$ and $426 \mathrm{keV}(9.88 \%)$ [11]. The $90 \%$ branch decays directly to the ground state of ${ }^{51} \mathrm{~V}$, and the $10 \%$ branch decays to the first excited state of ${ }^{51} \mathrm{~V}$, which promptly decays with the emission of a $320 \mathrm{keV}$ gamma ray to the ground state.

The chromium used for this experiment was originally in the form of $93 \%$ enriched ${ }^{50} \mathrm{Cr}_{2} \mathrm{O}_{3}[12,13]$. Achieving high enrichment was an important consideration since it significantly increases the specific activity of the source [14]. The Cr was converted to metal, chemically purified, formed into rods, and placed in two special moderator assemblies for irradiation at the BN-350 fast neutron reactor in Aktau, Kazakhstan [15]. The reactor power was set at $520 \mathrm{MW}$ for 90 days, followed by an increase to $620 \mathrm{MW}$ for 15 days. The source assemblies were removed from the reactor on 18 December 1994. The $\mathrm{Cr}$ rods, a total mass of $512.7 \mathrm{~g}$, were then removed from the moderator assemblies, cleaned, and placed in a tungsten shield $140 \mathrm{~mm}$ high $\times 80 \mathrm{~mm}$ diameter. This shield was 
sealed in a stainless steel outer casing and arrived at the Baksan Neutrino Observatory on 26 December 1994.

The 55 tonnes of Ga metal in SAGE is distributed among eight chemical reactors with approximately 7 tonnes in each. Each reactor contains the necessary equipment for Ge extraction. An identical reactor without extraction equipment was used for the source exposures. 13.1 tonnes of $\mathrm{Ga}$ was transferred into this irradiation vessel by a Teflon membrane pump from two of the reactors used for solar neutrino measurements. To start an irradiation, the ${ }^{51} \mathrm{Cr}$ source was placed inside a $\mathrm{Zr}$ reentrant tube at the center of this $\mathrm{Ga}$ target. Irradiations were ended by removing the source and pumping the $\mathrm{Ga}$ back to the original two reactors, where the Ge was extracted.

Eight irradiations were conducted between 26 December 1994 and 24 May 1995. The exposure periods for the first five measurements were chosen so each would have approximately equal statistical sensitivity, and a final three measurements were made at monthly intervals. The reference time at which the first irradiation began is denoted here SOB, for start of bombardment.

The tungsten shield around the source absorbed all but about 1 part in $10^{5}$ of the source activity. It was thus possible to determine the source strength by measuring its heat output with a specially developed calorimeter [16]. The source was removed from the irradiation vessel and placed in this calorimeter after each of the first seven exposures. The weighted average of these source power measurements is $112.3 \pm 0.8 \mathrm{~W}$ at SOB. The calorimeter was calibrated with electroheaters several times during the course of these measurements. The best fit half life to the power measurements is $28.03 \pm 0.23$ days, in reasonable agreement with the known ${ }^{51} \mathrm{Cr}$ half life of 27.702 days [17].

Using the power to activity conversion constant of $4.600 \pm 0.030 \mathrm{kCi} / \mathrm{W}$ implies the source strength was $516.6 \pm 6.0 \mathrm{kCi}$ at $\mathrm{SOB}$. The major uncertainty in this activity is the statistical uncertainty of $0.71 \%$ in the power measurements. Four systematic uncertainties have been added to this in quadrature: $0.65 \%$ from the conversion constant, $0.6 \%$ from different thermalization times between the source and the calibration heaters, and $0.2 \%$ from the $0.02 \%$ uncertainty in the ${ }^{51} \mathrm{Cr}$ half life. The final systematic uncertainty is from radioactive impurities in the irradiated $\mathrm{Cr}$ which can also produce heat in the calorimeter. The impurity content was determined during the $\mathrm{Ga}$ exposures by measuring gamma-ray spectra of the source with a $\mathrm{Ge}$ detector. The largest single contribution was $1.5 \mathrm{Ci}$ of ${ }^{46} \mathrm{Sc}$, and the total activity of all contaminants at SOB was estimated to be less than $2 \mathrm{Ci}$. Taking into account their half life and decay energy, an upper limit on the uncertainty in the source activity from impurities is $0.14 \%$.

The major uncertainty in the power to activity conversion constant is in the average energy released in ${ }^{51} \mathrm{Cr}$ decay, which we take as $36.671 \pm 0.190 \mathrm{keV} /$ decay. This value differs slightly from that used by Ref. [18] in that we have included, but Ref. [18] ignored, the contribu- tion of internal bremsstrahlung, which adds approximately $96 \mathrm{eV} /$ decay to the average, and that we use a branching fraction to the $320 \mathrm{keV}$ level of 0.0988 [11], whereas Ref. [18] used 0.0986.

The production rate of ${ }^{71} \mathrm{Ge}$ by a source whose strength is $A$ (MCi) is given by $p=\kappa\langle L\rangle A$ (atoms produced/day). The first term, $\kappa$, contains all the physical factors, such as neutrino interaction cross section, $\mathrm{Ga}$ density, etc., and has the value 0.3974 (neutrino captures/cm day MCi). For the absorption cross section we used the value given by Bahcall and Ulrich of $59.2 \times 10^{-46}(1.0 \pm$ $0.1) \mathrm{cm}^{2} /\left({ }^{71} \mathrm{Ga}-{ }^{51} \mathrm{Cr}\right.$ decay $)$, where the uncertainty is quoted at the 3 sigma level [19]. The second factor, $\langle L\rangle$, is the average neutrino path length through the Ga. The irradiation vessel was nearly cylindrical, but the bottom was dished. Monte Carlo integration using an accurate map of the vessel shape gives $\langle L\rangle=72.6 \pm 0.2 \mathrm{~cm}$. Combining these factors gives an expected production rate at SOB of $14.90 \pm 0.18$ atoms/day, where the uncertainties have been added in quadrature. This high rate is because of the small size of our source and the use of Ga metal as the target.

Recently, there was a reexamination of the absorption cross section by Hata and Haxton [20], with emphasis on the predicted contributions from excited states in ${ }^{71} \mathrm{Ge}$. This new analysis casts some doubt on the mean and corresponding uncertainty of the cross section. In our uncertainty estimate for the production rate above, we did not include the $3.3 \%$ (or 0.49 atoms/day) uncertainty of the Bahcall and Ulrich calculation. Hata and Haxton conclude that the previous estimate of the excited state cross section, inferred from $(p, n)$ reactions, was unjustified and only weakly constrained by previous experimental data. As a result, they assert that the neutrino absorption cross section is not known at the level of accuracy of the $\mathrm{Cr}$ source experiments, and it is these experiments themselves that best determine this cross section.

The experimental techniques for the $\mathrm{Cr}$ experiment were the same as used for solar neutrino measurements [21] and are only very briefly described here. After each $\mathrm{Cr}$ source exposure, the ${ }^{71} \mathrm{Ge}$ was chemically extracted, along with stable Ge carrier added prior to the start of exposure. This Ge was then synthesized into germane gas, mixed with $\mathrm{Xe}$, and placed in a small proportional counter where decays were observed for a period of six months. The electron capture decay of ${ }^{71} \mathrm{Ge}$ mainly gives Auger electrons which we detect at $10.4 \mathrm{keV}$ ( $K$ peak) or $1.2 \mathrm{keV}(L$ peak). The track length of these low-energy electrons in the counter gas is very short, producing a fast rising pulse. Background events, such as are produced by minimum ionizing particles, may have a similar energy, but will usually have longer path lengths and hence slower rise times.

We determine the pulse rise time with a method specially developed for SAGE. Wave forms that have been recorded with a one $\mathrm{GHz}$ digitizer are fit with an analytical function that describes the shape of the extended pulse in terms of the radial extent of the 
trajectory in the counter [22]. This method has great power in differentiating ${ }^{71} \mathrm{Ge}$ signals from background events, especially in the low-energy $L$-peak region. The digitizer began to be used in SAGE in late 1992. The solar neutrino results that we have published up to the present time have relied on a hardware-based risetime measurement of the amplitude of the differentiated counter pulse (ADP) but analysis of these data using the wave form analysis technique is in progress.

Our standard energy, rise time, and Rn cuts were applied to the counting data, and the time structure of the remaining candidate ${ }^{71} \mathrm{Ge}$ events was analyzed with a maximum likelihood method [23] to separate the ${ }^{71} \mathrm{Ge} 11.4$ day decay from a constant rate background. The only differences between this analysis and that done for the solar neutrino runs are that one must account for the decay of the ${ }^{51} \mathrm{Cr}$ during the period of exposure, include a "background" contribution from solar neutrinos, and make a carryover correction arising from the ${ }^{71} \mathrm{Ge}$ that was not removed because of the approximately $15 \%$ inefficiency of the preceding chemical extraction. Using the traditional notation of Cleveland [23], the likelihood function $(L)$ is given by

$$
L=\exp \left(-b T_{L}+a \Delta / \lambda_{71}\right) \prod_{i=1}^{N}\left[b+a \exp \left(-\lambda_{71} t_{i}\right)\right],
$$

where $b$ is the background rate, $T_{L}$ is the live time of counting, $\lambda_{71}$ is the ${ }^{71} \mathrm{Ge}$ decay constant, $\Delta$ is the integral over the live time of $\exp \left(-\lambda_{71} t\right)$, and $t_{i}$ are the times of occurrence of the $N$ candidate events. The parameter $a$ contains contributions from the three separate processes (source, solar, and carryover) that produce ${ }^{71} \mathrm{Ge}$, i.e., $a=a_{\mathrm{Cr}}+a_{0}+a_{\mathrm{co}}$, where for extraction $k$

$$
\begin{aligned}
a_{\mathrm{Cr}}^{k}= & p_{\mathrm{Cr}} \exp \left[-\lambda_{51}\left(t_{s}^{k}-T\right)\right] \\
& \times \varepsilon^{k}\left[\exp \left(-\lambda_{51} \theta_{\mathrm{Cr}}^{k}\right)\right. \\
& \left.-\exp \left(-\lambda_{71} \theta_{\mathrm{Cr}}^{k}\right)\right] /\left(1-\lambda_{51} / \lambda_{71}\right), \\
a_{0}^{k}= & p_{0} \varepsilon^{k}\left[1-\exp \left(-\lambda_{71} \theta_{0}^{k}\right)\right],
\end{aligned}
$$

$$
a_{\mathrm{co}}^{k}=a^{k-1}\left(\varepsilon^{k} / \varepsilon^{k-1}\right) \exp \left(-\lambda_{71} \theta_{0}^{k}\right)\left(1-\varepsilon_{\mathrm{Ga}}^{k-1}\right) .
$$

Here $p_{\mathrm{Cr}}$ and $p_{0}$ are the rates of production of ${ }^{71} \mathrm{Ge}$ by the ${ }^{51} \mathrm{Cr}$ source and solar neutrinos, respectively; $\lambda_{51}$ is the decay constant of ${ }^{51} \mathrm{Cr}$; $t_{s}$ is the starting time of each source exposure; $T$ is the reference time $\mathrm{SOB} ; \theta_{\mathrm{Cr}}$ and $\theta_{0}$ are the times of exposure of the $\mathrm{Ga}$ to the ${ }^{51} \mathrm{Cr}$ source and to solar neutrinos, respectively; $\varepsilon$ is the product of extraction and counting efficiencies; and $\left(1-\varepsilon_{\mathrm{Ga}}\right)$ is the inefficiency of extraction of $\mathrm{Ge}$ from the Ga. With these definitions, as the source decays, its derived strength $p_{\mathrm{Cr}}$ is automatically referred to time $T$. Maximization of the product of the likelihood functions for all runs yields the global production rate $p_{\mathrm{Cr}}$ and individual background rates for the $K$ and $L$ peaks in each run. The solar production rate $p_{0}$ was fixed at 0.27 /day, the rate corresponding to $69 \mathrm{SNU}$ [2] on 13.1 tonnes of Ga.

The results of the data analysis are given in Table I. The combined fit to the eight extractions considering only the $L$ peak ( $K$ peak) gives production rates of $16.8_{-2.4}^{+2.6} /$ day $\left(12.4_{-1.8}^{+2.0} /\right.$ day $)$. The result considering both the $K$ and $L$ peaks is $14.2_{-1.5}^{+1.6} /$ day. Here the uncertainties are all statistical. This is the largest production rate ever measured from a low-energy neutrino source and is 50 times higher than the rate from solar neutrinos. A fit permitting the ${ }^{71} \mathrm{Ge}$ half life to vary gives $13.8 \pm$ 2.0 days, compared with its known half life of 11.4 days. Our solar neutrino results in the past have relied on the ADP measurement of the pulse rise time, which gives good background discrimination only in the $K$ peak. A combined fit to the eight source extractions that uses events in the $K$ peak selected by ADP gives a rate of $11.2_{-1.5}^{+1.7} /$ day, similar to the result of 12.4 /day that is obtained when wave form analysis is used for event selection. The Smirnov-Cramer-Von Mises parameter

\begin{tabular}{|c|c|c|c|c|c|c|c|c|}
\hline $\begin{array}{l}\text { Exposure } \\
\text { reference } \\
\text { name }\end{array}$ & $\begin{array}{l}\text { Source } \\
\text { strength } \\
(\mathrm{kCi})\end{array}$ & $\begin{array}{l}\text { Number } \\
\text { of } \\
\text { candidate } \\
\text { events }\end{array}$ & $\begin{array}{c}\text { Number } \\
\text { fit to } \\
{ }^{71} \mathrm{Ge}\end{array}$ & $\begin{array}{c}\text { Number } \\
\text { assigned } \\
\text { to solar } \\
\text { neutrinos }\end{array}$ & $\begin{array}{l}\text { Number } \\
\text { assigned } \\
\text { to } \\
\text { carryover }\end{array}$ & $\begin{array}{c}{ }^{71} \mathrm{Ge} \\
\text { prod. rate } \\
\text { by } \mathrm{Cr} \\
\text { source } \\
\text { (/day) }\end{array}$ & $\mathrm{Nw}^{2}$ & Prob. \\
\hline $\mathrm{Cr} 1$ & 516.1 & 47 & 38.7 & 0.9 & 0 & $23.2_{-4.0}^{+4.3}$ & 0.151 & $17 \%$ \\
\hline $\mathrm{Cr} 2$ & 434.5 & 39 & 23.3 & 0.7 & 2.5 & $12.2_{-3.5}^{+3.8}$ & 0.169 & $9 \%$ \\
\hline $\mathrm{Cr} 4$ & 288.0 & 36 & 25.2 & 1.2 & 1.4 & $11.6_{-2.7}^{+3.1}$ & 0.060 & $63 \%$ \\
\hline Cr 5 & 194.0 & 33 & 13.8 & 1.8 & 0 & $8.8_{-3.4}^{+3.9}$ & 0.042 & $68 \%$ \\
\hline Cr 6 & 97.7 & 48 & 5.9 & 1.0 & 0.4 & $9.8_{-7.5}^{+9.0}$ & 0.039 & $85 \%$ \\
\hline $\mathrm{Cr} 7$ & 50.1 & 27 & 4.3 & 1.7 & 0 & $7.8_{-7.8}^{+10.8}$ & 0.058 & $67 \%$ \\
\hline Cr 8 & 23.7 & 21 & 4.4 & 1.4 & 0 & $20.4_{-18.3}^{+23.3}$ & 0.034 & $86 \%$ \\
\hline
\end{tabular}
[24], $\mathrm{Nw}^{2}$, given in Table I provides a measure of the goodness of fit. The probability values were determined by Monte Carlo methods.

TABLE I. $K+L$ peak counting results from the wave form analysis. The production rate for each exposure has been normalized to the starting time of the first exposure. 
The overall systematic uncertainty can, for the most part, be determined in a manner similar to what we have presented for the solar runs [2]. The chemical extraction efficiency is typically $80 \%$ with an uncertainty of $\pm 4.1 \%$. The volume efficiency of all counters used for these extractions was directly measured by filling each with an $\mathrm{Ar}-\mathrm{CH}_{4}$ mixture to which a small quantity of ${ }^{37} \mathrm{Ar}$ was added. The resultant uncertainty in the counting efficiency, including uncertainties in setting energy and rise time windows for event selection, is $+3.2 \%,-5.9 \%$. Some signal from Rn may still be present due to the inefficiency of the time cuts, and this contribution is estimated to be $-0.7 \%$. The uncertainty in the solar neutrino rate $p_{0}$ results in a $1.1 \%$ uncertainty in $p_{\mathrm{Cr}}$. The total number of estimated carryover events is 4.3 with a $10 \%$ uncertainty, which implies an uncertainty in $p_{\mathrm{Cr}}$ of $0.3 \%$. Combining all these contributions yields an overall systematic uncertainty in $p_{\mathrm{Cr}}$ of $+5.3 \%,-7.3 \%$. Thus the measured rate, $p_{\mathrm{Cr}}$, is $14.2_{-1.5}^{+1.6}$ (stat) $+0.8 /$ -1.0 (syst)/day.

The ratio $(R)$ of the measured ${ }^{71} \mathrm{Ge}$ production rate to the rate expected from the source strength is

$$
R=\frac{\left(p_{\mathrm{Cr}}\right)_{\text {measured }}}{\left(p_{\mathrm{Cr}}\right)_{\text {expected }}}=0.95 \pm 0.12,
$$

where the systematic and statistical uncertainties have been added in quadrature. This result shows that the total efficiency of the SAGE experiment to the neutrinos from ${ }^{51} \mathrm{Cr}$ is very close to $100 \%$.

Since the neutrino spectrum from ${ }^{51} \mathrm{Cr}$ differs from the solar spectrum and the total experimental efficiency for each solar neutrino measurement is known to a higher precision than the $12 \%$ uncertainty obtained with the ${ }^{51} \mathrm{Cr}$ source experiment, the solar neutrino measurement should not be scaled by the above ratio.

GALLEX has also made ${ }^{51} \mathrm{Cr}$ neutrino source measurements with the result $R=0.92 \pm 0.08$ [5]. Thus both SAGE and GALLEX, which employ very different chemistries, give similar solar neutrino results, and have verified their efficiencies with neutrino source measurements. The solar neutrino capture rate measured by the $\mathrm{Ga}$ experiments is in striking disagreement with standard solar model predictions, and there is considerable evidence that this disagreement is not an experimental artifact.

We thank E. N. Alexeyev, J. Bahcall, M. Baldo-Ceolin, L. B. Bezrukov, S. Brice, A.E. Chudakov, G.T. Garvey, W. Haxton, P. M. Ivanov, V. V. Kuzminov, V.A. Matveev, V.A. Rubakov, R. G.H. Robertson, and A.N. Tavkhelidze for stimulating our interest and for fruitful discussions. We acknowledge the support of the Russian Academy of Sciences, the Institute for Nuclear Research of the Russian Academy of Sciences, the Russian Ministry of Science and Technology, the Russian Foundation of Fundamental Research, the Division of Nuclear Physics of the U.S. Department of Energy, and the U.S. National Science Foundation. This research was made possible in part by Grant No. M7F000 from the International Science
Foundation and Grant No. M7F300 from the International Science Foundation and the Russian Government.

*Present address: Institute of Theoretical and Experimental Physics, 117259 Moscow, Russia.

†Present address: National Institute of Standards and Technology, Bldg. 235/A106, Gaithersburg, MD 20899.

${ }^{\ddagger}$ Present address: Department of Particle and Nuclear Physics, Oxford University, Keble Road, Oxford OX1 3RH, U.K.

[1] G. Audi and A. H. Wapstra, Nucl. Phys. A595, 409 (1995).

[2] J. N. Abdurashitov et al., Phys. Lett. B 328, 234 (1994); S. R. Elliott et al., in Proceedings of the XXXth Rencontres de Moriond, Electroweak Interactions and Unified Theories, Les Arcs, Savoie, France, 1995, edited by J. Tran Thanh Van (Editions Frontieres, Singapore, 1995), p. 439.

[3] J. N. Bahcall, M. Pinsonneault, and G. J. Wasserburg, Rev. Mod. Phys. 67, 781 (1995).

[4] S. Turck-Chieze and I. Lopes, Astrophys. J. 408, 347 (1993).

[5] W. Hampel et al. (to be published).

[6] B. T. Cleveland et al., Nucl. Phys. B38, 47 (1995).

[7] Y. Suzuki et al., Nucl. Phys. B38, 54 (1995).

[8] J. N. Bahcall et al., Nature (London) 375, 29 (1995).

[9] V.A. Kuzmin, Ph.D. thesis, Lebedev Physics Institute, Moscow, 1967.

[10] R.S. Raghavan, Brookhaven National Laboratory Report No. 50879, 1978, Vol. 2.

[11] Table of Isotopes, edited by V.S. Shirley (John Wiley and Sons, New York, 1996), 8th ed., p. 209.

[12] A. Tikhomirov, Nucl. Instrum. Methods Phys. Res., Sect. B 70, 1 (1992).

[13] G. E. Popov et al., Nucl. Instrum. Methods Phys. Res., Sect. A 362, 532 (1995).

[14] V. N. Gavrin, S. N. Dan'shin, G. T. Zatsepin, and A. V. Kopylov, Report No. INR P-335, 1984, in Russian.

[15] A. V. Zvonarev et al., At. Energ. 80, 107 (1996).

[16] I. N. Belousov et al., in Proceedings of the International School on Particles and Cosmology, Baksan Valley, Russia, 1991, edited by V. A. Matveev, E. N. Alexeyev, V. A. Rubakov, and I. I. Tkachev (World Scientific Publishing, Singapore), p. 59.

[17] C. Zhou, Nucl. Data Sheets 63, 229 (1991).

[18] P. Anselmann et al., Phys. Lett. B 342, 440 (1995).

[19] J. N. Bahcall and R. K. Ulrich, Rev. Mod. Phys. 60, 297 (1988).

[20] N. Hata and W. Haxton, Phys. Lett. B 353, 422 (1995).

[21] The only modification from the standard extraction procedures that was used for the $\mathrm{Cr}$ experiment was the transfer of $\mathrm{Ga}$ from two reactors to the irradiation vessel and back. To verify that this transfer process did not gain or lose ${ }^{71} \mathrm{Ge}$ activity, during the course of usual solar neutrino measurements, a set of two-reactor experiments was made in which this Ga transfer was included. The measured production rate in the two reactors was consistent with that from solar neutrinos.

[22] S. R. Elliott, Nucl. Instrum. Methods Phys. Res., Sect. A 290, 158 (1990).

[23] B. T. Cleveland, Nucl. Instrum. Methods Phys. Res. 214, 451 (1983).

[24] A. W. Marshall, Ann. Math. Stat. 29, 307 (1958). 